\title{
CALCULATION OF THE FREQUENCY RESPONSE AND BANDWIDTH IN LOW NUMERICAL APERTURE STEP-INDEX PLASTIC OPTICAL FIBER USING TIME- DEPENDENT POWER FLOW EQUATION
}

\author{
Branko Drljača $^{1 *}$, Slavica Jovanović ${ }^{1}$, Svetislav Savović ${ }^{2}$ \\ ${ }^{1}$ Faculty of Natural Sciences, University of Priština, Kosovska Mitrovica, Serbia. \\ ${ }^{2}$ Faculty of Science, University of Kragujevac, Kragujevac, Serbia.
}

\begin{abstract}
Time-dependent power flow equation is employed to calculate frequency response and bandwidth of low numerical aperture step-index optical fiber excited with Gaussian-like light source with large width. Both, frequency response and

bandwidth, are specified as function of the fiber length measured from the input end of the fiber. Analytical and numerical solutions are compared and good agreement between results is obtained.
\end{abstract}

Key words: Plastic optical fiber, Frequency response, Bandwidth.

\section{INTRODUCTION}

In recent decades there has been significant growth in the amount of transferred data both in long-distance and short-distance communication. For highperformance short-distance communication, such as local-area networks (LANs), multi-node bus networks plastic or digital car networks, industrial control, plastic optical fibers (POFs) are often considered as best choice ((Golowich et al., 2003), (Ishigure et al., 2000), (Green, 1996), (Koeppen et al., 1998)). This is based upon their low cost, ease of manipulation and high bandwidth.

In comparison to systems based upon glass optical fiber or copper wire, systems based upon POFs are much more affordable in total. With a fiber diameter of 1 millimeter, and a core diameter of $980 \mu \mathrm{m}$ (for most applications) cutting and treating of POF's ends is done without much effort. Nonetheless large core diameter allows POFs to be paired with light sources of higher NA, such as LED sources, using lowprecision plastic couplers. This, in total, results in inexpensive but robust systems that are easy to interconnect. Bandwidth of typical step-index optical fiber (SI POF) is higher than with copper wire systems and approximately is $100 \mathrm{MHz}$ over $100 \mathrm{~m}$ (Koike, 2015). Bit rates of about $200 \mathrm{Mbit} / \mathrm{s}$ over $50 \mathrm{~m}$ can be obtained without any additional measures, and bit rates of over $1000 \mathrm{Mbit} / \mathrm{s}$ over $50 \mathrm{~m}$ have been realized with special transmission schemes (equalizing, OFDM) over the last years.
From the previous statements it is clear that transmission properties of SI POF, such as bandwidth and frequency response, have most significant role in their further development. Frequency response and consequently bandwidth of multimode SI POFs depend strongly on the mode-dependent attenuation, modal dispersion and the rate of mode coupling (power transfer from lower to higher order modes). In order to examine influence of the width of launch beam to frequency response and bandwidth we calculated it for tested SI POF and compared with available results for the same fiber (Savovic et al., 2014a)

This paper is organized as follows. In chapter 2 mathematical model based upon time-dependent power flow equation, developed by Gloge (Gloge, 1973), is presented. Using the time-dependent power flow equation we have determined frequency response and bandwidth of SI POF with low numerical aperture in addition to mode coupling and mode-dependent attenuation. Comparison of results obtained by analytical and numerical solution is presented in chapter 3 . Finally conclusion is presented in chapter 4 .

\section{TIME-DEPENDENT POWER FLOW EQUATION}

\subsection{Analytical solution}

Gloge's time-dependent power flow equation can be written as (Gloge, 1973): 


$$
\begin{aligned}
\frac{\partial P(\theta, z, t)}{\partial z} & +\frac{\partial t}{\partial z} \frac{\partial P(\theta, z, t)}{\partial t}=-\alpha(\theta) P(\theta, z, t)+ \\
& +\frac{1}{\theta} \frac{\partial}{\partial \theta}\left[\theta D(\theta) \frac{\partial P(\theta, z, t)}{\partial \theta}\right]
\end{aligned}
$$

where $P(\theta, z, t)$ is power distribution over angle $\theta$ measured with respect to fiber axis, space $z$ and time $t$; $\alpha(\theta)$ is mode-dependent attenuation; $\partial t / \partial z$ is mode delay per unit length; and $D(\theta)$ is the modedependent coupling coefficient. Mode-dependent attenuation can be written in the form $\alpha(\theta)=\alpha_{0}+A \theta^{2}+\ldots \quad$ Term $\quad \alpha_{0}$ represents loss common to all modes and it can be accounted for by multiplying the end-solution by $e^{-\alpha_{0} z}$ (Gloge, 1973). More important is the term $A \theta^{2}$ which describes the losses at core-cladding boundary and for that reason, in solving (1), one needs to consider only the term $A \theta^{2}$ as the most dominant of the higher order modes (Gloge, 1972). Coupling coefficient $D(\theta)$ is, as it is stated, also mode-dependent (Olshansky, 1975), but mode-independent coupling constant $D$ has been used frequently by other authors ((Gloge, 1973), (Drljaca et al., 2009), (Drljaca et al., 2012)). If coupling constant $D$ is used equation (1) can be written as (Gloge, 1973):

$$
\begin{aligned}
\frac{\partial P(\theta, z, t)}{\partial z} & =-A \theta^{2} P(\theta, z, t)-\frac{n}{2 c} \theta^{2} \frac{\partial P(\theta, z, t)}{\partial t}+ \\
& +\frac{D}{\theta} \frac{\partial}{\partial \theta}\left(\theta \frac{\partial P(\theta, z, t)}{\partial \theta}\right) .
\end{aligned}
$$

In order to solve time-dependent equation (2) analytically it is transformed into time-independent equation (Gloge, 1973):

$$
\frac{\partial p}{\partial z}=-A \sigma^{2} \theta^{2} p+\frac{D}{\theta} \frac{\partial}{\partial \theta}\left(\theta \frac{\partial p}{\partial \theta}\right) .
$$

Using time-independent power flow equation (3) analytical solution for impulse response can be obtained (Gloge, 1973). For short fibres $\left(\mathrm{z}<<1 /\left(2(A D)^{1 / 2}\right)\right)$, impulse response is:

$$
Q(z, t)=\frac{2 c \pi}{n z\left(1+\gamma_{\infty} z\right)} \exp \left(-2 c t / n \Theta_{0}^{2} z\right),
$$

while in case of long fibers $\left(\left(\mathrm{z}>>1 /\left(2(A D)^{1 / 2}\right)\right)\right.$, it has the form:

$$
Q(z, t)=\Theta_{0}^{2} \sqrt{\frac{\pi}{T t}}\left(\frac{t}{\gamma_{\infty} z T}+\frac{1}{2}\right)^{-1} \exp \left(-\frac{\gamma_{\infty}^{2} z^{2} T}{4 t}-\frac{t}{T}\right)
$$

After obtaining impulse response, frequency response can be obtained easily by applying Fourier transform to the impulse response:

$$
H(f)=\int_{-\infty}^{\infty} Q(t) \exp (2 \pi i f t) d t
$$

After obtaining frequency response, $-3 \mathrm{~dB}$ bandwidth, for different lengths, is easily obtained by selecting frequencies for which frequency response has $50 \%$ drop for given length.

\subsection{Numerical solution}

In order to solve time-dependent power flow equation numerically we start from equation (1). In the same manner, as with analytical solution, for modedependent attenuation term $A \theta^{2}$ is used and coupling coefficient is once more assumed to be constant $-D$. If so, equation (1) can be written as (2). After applying the Fourier transform to (2):

$$
p(\theta, z, \omega)=\int_{-\infty}^{\infty} P(\theta, z, t) e^{-j \omega t} d t
$$

the time-dependent equation (2) transforms into (8):

$$
\begin{aligned}
\frac{\partial p(\theta, z, \omega)}{\partial z} & =-\left[A \theta^{2}+j \omega \frac{n}{2 c} \theta^{2}\right] p(\theta, z, \omega)+ \\
& +\frac{D}{\theta} \frac{\partial p(\theta, z, \omega)}{\partial \theta}+D\left(\theta \frac{\partial^{2} p(\theta, z, \omega)}{\partial \theta^{2}}\right)
\end{aligned}
$$

where $\omega=2 \pi f$ is the angular frequency.

The boundary conditions are:

$$
p\left(\theta_{c}, z, \omega\right)=0,\left.\quad D \frac{\partial p(\theta, z, \omega)}{\partial \theta}\right|_{\theta=0}=0,
$$

where $\theta_{c}$ is the critical angle. The first condition implies that modes with infinitely large loss do not transfer any power; the second condition implies that mode coupling is limited to modes travelling with the angle $\theta>0$.

Since $p(\theta, z, \omega)$ is complex we can therefore separate $p(\theta, z, \omega)$ into its real and imaginary parts, $p=p^{r}+j p^{i}$. Equation (8) can now be rewritten as the following system of simultaneous differential equations: 


$$
\begin{aligned}
& \frac{\partial p^{r}}{\partial z}=-A \theta^{2} p^{r}+\frac{D}{\theta} \frac{\partial p^{r}}{\partial \theta}+D \frac{\partial^{2} p^{r}}{\partial \theta^{2}}+\omega \frac{n}{2 c} \theta^{2} p^{i} \\
& \frac{\partial p^{i}}{\partial z}=-A \theta^{2} p^{i}+\frac{D}{\theta} \frac{\partial p^{i}}{\partial \theta}+D \frac{\partial^{2} p^{i}}{\partial \theta^{2}}-\omega \frac{n}{2 c} \theta^{2} p^{r}
\end{aligned}
$$

After obtaining $p^{r}$ and $p^{i}$ by solving equations (10), frequency response at distance $z$ from input end of the fiber is calculated as:

$$
H(z, \omega)=\frac{2 \pi \int_{0}^{\theta_{c}} \theta\left[p^{r}(\theta, z, \omega)+j p^{i}(\theta, z, \omega)\right] d \theta}{2 \pi \int_{0}^{\theta_{c}} \theta\left[p^{r}(\theta, 0, \omega)+j p^{i}(\theta, 0, \omega)\right] d \theta}
$$

After separating the power flow equation (8) into two simultaneous equations (10), we solved the latter (10) by explicit finite difference method (EFDM). Using central difference scheme for derivatives $\partial p(\theta, z, \omega) / \partial \theta$ and $\partial^{2} p(\theta, z, \omega) / \partial \theta^{2}$ (Savovic et al. 2004), (Djordjevich et al. 2000), (Anderson 1995):

$$
\begin{aligned}
& \left(\frac{\partial p(\theta, z, \omega)}{\partial \theta}\right)_{k, l}=\frac{p_{k+1, l}-p_{k-1, l}}{2 \Delta \theta}+O(\Delta \theta)^{2} \\
& \left(\frac{\partial^{2} p(\theta, z, \omega)}{\partial \theta^{2}}\right)_{k, l}=\frac{p_{k+1, l}-2 p_{k, l}+p_{k-1, l}}{(\Delta \theta)^{2}}+O(\Delta \theta)^{2}
\end{aligned}
$$

and using the forward difference scheme for the derivative $\partial p(\theta, z, \omega) / \partial z$ :

$$
\left(\frac{\partial p(\theta, z, \omega)}{\partial z}\right)_{k, l}=\frac{p_{k, l+1}-p_{k, l}}{\Delta z}+O(\Delta z)
$$

equations (10) can be written in the form:

$$
\begin{aligned}
& p_{k, l+1}^{r}= \\
& =\left(\frac{\Delta z D}{\Delta \theta^{2}}-\frac{\Delta z D}{2 \theta_{k} \Delta \theta}\right) p_{k-1, l}^{r}+\left(1-\frac{2 \Delta z D}{\Delta \theta^{2}}-\Delta z A \theta_{k}^{2}\right) p_{k, l}^{r}+ \\
& +\left(\frac{\Delta z D}{2 \theta_{k} \Delta \theta}+\frac{\Delta z D}{\Delta \theta^{2}}\right) p_{k+1, l}^{r}+\frac{\omega n \Delta z}{2 c} \theta_{k}^{2} p_{k, l}^{i}
\end{aligned}
$$

and

$$
\begin{aligned}
& p_{k, l+1}^{i}= \\
& =\left(\frac{\Delta z D}{\Delta \theta^{2}}-\frac{\Delta z D}{2 \theta_{k} \Delta \theta}\right) p_{k-1, l}^{i}+\left(1-\frac{2 \Delta z D}{\Delta \theta^{2}}-\Delta z A \theta_{k}^{2}\right) p_{k, l}^{i}+
\end{aligned}
$$

$+\left(\frac{\Delta z D}{2 \theta_{k} \Delta \theta}+\frac{\Delta z D}{\Delta \theta^{2}}\right) p_{k+1, l}^{i}-\frac{\omega n \Delta z}{2 c} \theta_{k}^{2} p_{k, l}^{r}$.

Boundary conditions (9) now become:

$$
\begin{aligned}
& p_{N, l}^{r}=0, p_{N, l}^{i}=0 \text { and } \\
& p_{0, l}^{r}=p_{1, l}^{r}, \quad p_{0, l}^{i}=p_{1, l}^{i}
\end{aligned}
$$

where $N=\theta_{c} / \Delta \theta$ is the grid size in the $\theta$ direction. In order to prevent the problem of singularity at grid points $\theta=0$, the following relation is used (Anderson 1995):

$$
\lim _{\theta \rightarrow 0} \frac{1}{\theta} \frac{\partial}{\partial \theta}\left(\theta \frac{\partial p}{\partial \theta}\right)=\left.2 \frac{\partial^{2} p}{\partial \theta^{2}}\right|_{\theta=0}
$$

In our previous work (Savovic et al. 2014a), we have determined frequency response and bandwidth of the low numerical aperture $(\mathrm{NA}=0.3)$ SI POF for a Dirac impulse in time and a laser mode distribution with the $\theta_{0}=0^{\circ}$ (central launch) and FWHM $=7.5^{\circ}$ in the parallel plane. In this work, using Gloge's analytical solution of (2) and our numerical results for (2) we calculate the frequency response and bandwidth of same SI POFs for a Dirac impulse in time and a laser mode distribution with the $\theta_{0}=0^{\circ}$ and FWHM $=16^{\circ}$ in the parallel plane and compare obtained analytical to numerical results.

\section{ANALYTICAL AND NUMERICAL RESULTS}

In this section we present results for frequency response and bandwidth of the tested MH4001 ESKA Mitsubishi Rayon fiber (MH fiber). The fiber`s dimensions are typical for this kind of fiber. Core and clad diameters are $d_{\text {core }}=980 \mu \mathrm{m}$ and $d_{\text {clad }}=1000 \mu \mathrm{m}$, respectively. Numerical aperture is $\mathrm{NA}=0.3$, core refractive index is $n=1.49$, and critical angle is $\theta_{c}=11.7^{\circ}$ (measured inside the fiber) or $\theta_{c}=17.6^{\circ}$ (measured in air). The number of modes in this stepindex multimode plastic optical fiber at $\lambda=660 \mathrm{~nm}$ is $N=2 \pi^{2} a^{2}(\mathrm{NA})^{2} / \lambda^{2} \approx 1.02 \times 10^{6}$, where $a$ is radius of the fiber core. This large number of modes may be represented by a continuum as required for application of equation (2).

In recently published works (Savovic et al. 2014a), (Savovic et al. 2014b), coupling coefficient $D$ and loss coefficient $\alpha(\theta) \quad\left(\alpha(\theta) \approx \alpha_{0}+A \theta^{2}\right)$ for this fiber were calculated as $D=1.62 \times 10^{-3} \mathrm{rad}^{2} / \mathrm{m}$ and $\alpha_{0}=0.10793$ 
$1 / \mathrm{m}$ and $A=0.29166\left(\mathrm{rad}^{2} \mathrm{~m}\right)^{-1}$. The lengths of the MH4001 fiber that we used in our calculations were 3 m, $5 \mathrm{~m}, 10 \mathrm{~m}, 15 \mathrm{~m}, 25 \mathrm{~m}, 50 \mathrm{~m}$ and $100 \mathrm{~m}$. A Dirac impulse in time and mode distribution with the $\theta_{0}=0^{\circ}$ (central launch) and FWHM $=16^{\circ}$ in the parallel plane were used for the input. Analytical and numerical results for the frequency response for different fiber lengths of the $\mathrm{MH}$ fiber are shown in Figure 1. Analytical and numerical results match well. A pronounced drop at low frequencies is apparent for long fiber lengths. Fig. 2 shows analytically and numerically obtained bandwidth for MH4001 fiber. Bandwidth is shown in log-log scale versus length of the fiber. Slightly lower values of bandwidth are observed than in the case of the narrower beam with $\mathrm{FWHM}=7.5^{\circ}$ previously investigated by (Savovic et al. 2014a). This is more pronounced for shorter fiber lengths and it is due to the excitation of higher order modes when fiber is excited with wider input light beam. Higher modes experience higher loss since at shorter fiber lengths dominant process is modedependent attenuation. With increasing fiber length mode coupling begins to significantly influence fibers frequency response and bandwidth. In that case influence of the width of the launched beam on the fiber bandwidth becomes negligible since process of mode coupling at those lengths is either finished or came near end.

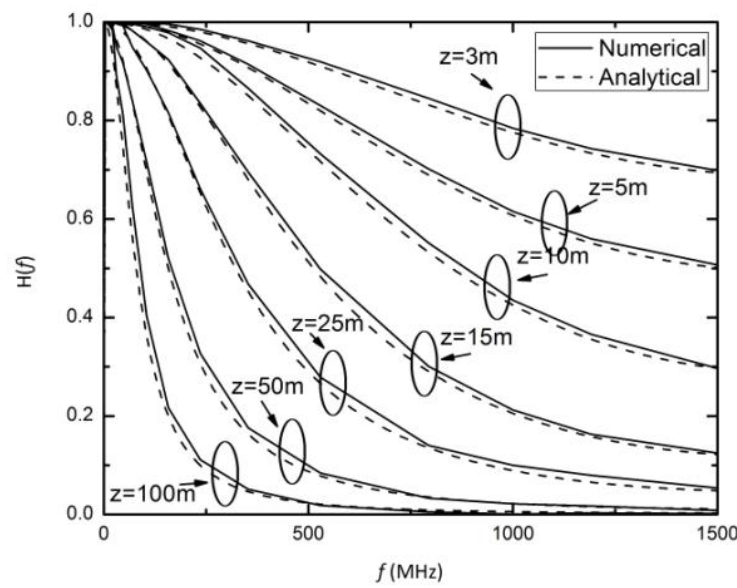

Fig. 1 Analytical (dashed line) and numerical (solid line) results for the frequency response for $\mathrm{MH}$ fiber, for different fiber lengths.



Fig. 2 Analytical (dashed line) and numerical (solid line) results for the bandwidth for $\mathrm{MH}$ fiber, for different fiber lengths.

\section{CONCLUSION}

Analytical and numerical solution of timedependent power flow equation were employed to calculate frequency response and bandwidth of MH4001 step-index optical fiber. Obtained results show good match. Moreover if our results are compared to previously obtained results for the same fiber, but different input conditions (narrower width of input light beam) (Savovic et al., 2014a), expected results are obtained. Namely, frequency response (and consequently bandwidth) obtained for the input light beam with $\mathrm{FWHM}=16^{\circ}$ (this paper) show slightly lower values than in the case of the narrower beam with FWHM $=7.5^{\circ}$ previously investigated by (Savovic et al. 2014a). This is more pronounced for shorter fiber lengths where mode-dependent attenuation is dominant process. With increasing fiber length mode coupling begins to significantly influence fibers transfer characteristics, that is frequency response and bandwidth. Namely, influence of the width of the launched beam on the fiber's frequency response and bandwidth, is more pronounced at shorter fiber lengths (Savovic et al., 2010), leading to lower values of frequency response and bandwidth and becomes negligible at longer fiber lengths.

\section{ACKNOWLEDGMENT}

The work described in this paper was supported by a grant from Serbian Ministry of Science and Technological Development (project no. 171011). 


\section{REFERENCES}

Anderson, J.D. 1995. Computational Dynamics.New York, USA: McGraw-Hill.

Djordjevich, A., \& Savović, S. 2000. Investigation of mode coupling in step index plastic optical fibers using the power flow equation. IEEE Photon. Technol. Lett., 12, pp. 1489-1491.

Drljača, B., Savović, S., \& Djordjevich, A. 2009. Calculation of the Impulse Response of Step-Index Plastic Optical Fibers Using the Time-Dependent Power Flow Equation. Acta Phys. Pol. A, 116, pp. 658-660.

Drljača, B., Djordjevich, A., \& Savović, S. 2012. Frequency response in step-index plastic optical fibers obtained by numerical solution of the timedependent power flow equation. Optics and Laser Technology, 44(6), pp. 1808-1812.

Gloge, D. 1973. Impulse Response of Clad Optical Multimode Fibers. Bell Syst. Tech. J., 52, pp. 801816.

Gloge, D. 1972. Optical power flow in multimode fibers. Bell Syst. Tech. J., 51, pp. 1767-1783.

Golowich, S.E., White, W., Reed, W.A., \& Knudsen, E. 2003. Quantitative estimates of mode coupling and differential modal attenuation in perfluorinated graded-index plastic optical fiber. Journal of Lightwave Technology, 21(1), pp. 111-121. doi:10.1109/JLT.2003.808668.

Green, P.E. 1996. Optical networking update. IEEE Journal on Selected Areas in

*E-mail: branko.drljaca@pr.ac.rs
Communications, 14(5),

pp.

764-779. doi:10.1109/49.510902.

Ishigure, T., Kano, M., \& Koike, Y. 2000. . J. Lightwave Technol, 18, pp. 959-965.

Koeppen, C., Shi, R.F., Chen, W.D., \& Garito, A.F. 1998. . J. Opt. Soc. Am. B, 15, pp. 727-739.

Koike, Y. 2015. Fundamentals of Plastic Optical Fibers.Weinheim, Germany: Wiley-VCH.

Olshansky, R. 1975. Mode Coupling Effects in Graded-index Optical Fibers. Appl Opt, 14(4), pp. 935-45. pmid:20135002.

Savović, S., \& Djordjevich, A. 2004. Influence of numerical aperture on mode coupling in step-index plastic optical fibers. Appl Opt, 43(29), pp. 5542-6. pmid:15508612.

Savović, S., Djordjevich, A., Tse, P.W., Zubia, J., Mateo, J., \& Losada, M.A. 2010. Determination of the width of the output angular power distribution in step index multimode optical fibers. J. Opt., 12(115405), p. 5.

Savović, S., Drljača, B., Kovačević, M.S., Djordjevich, A., Bajić, J.S., Stupar, D.Z., \& Stepniak, G. 2014. Frequency response and bandwidth in low-numerical-aperture step-index plastic optical fibers. Appl Opt, 53(30), pp. 69997003. pmid:25402786.

Savović, S., Kovačević, M.S., Djordjevich, A., Bajić, J.S., Stupar, D.Z., \& Stepniak, G. 2014. Mode coupling in low NA plastic optical fibers,. Opt. Laser Technol, 60, pp. 85-89. 